\title{
Gestational Diabetes Mellitus: Epigenetic Changes A Challenge
}

\author{
R. P.Kalsait ${ }^{1}$, A. R. Kalsait ${ }^{2}$, R. Khetade ${ }^{1}$, R. Ambad ${ }^{3}$ and N Bankar ${ }^{4}$ \\ ${ }^{1}$ Nagpur College of Pharmacy, Wanadongri, Hingna Road, Nagpur \\ ${ }^{2}$ Lata Medical and Research Foundation, Vasant Nagar, Nagpur \\ ${ }^{3}$ Associate Professor Datta Meghe Medical Sciences, Shalinitai Meghe Hospital and \\ Research Centre, Wanadongri, Nagpur, India \\ ${ }^{4}$ Associate Professor Deprtment of Microbiology Jawaharlal Nehru Medical College, \\ Datta Meghe Institute of Medical Sciences Sawangi (Meghe) Wardha
}

\section{ABSTRACT}

Around 7\% of pregnancies suffered with the Gestational Diabetes Mellitus (GDM), this number may vary considerably depending upon the demographic characteristics of population. GDM increases risk to offspring like obesity and type II DM, hypothesis suggest that epigenetic changes has an effect of GDM on offspring and could provide an a data to reduce such risk in next generation. Epigenetics involves the group of molecular mechanisms which includes DNA methylation, modification on histone (methylation, acetylation, crotonylation, Ubiquitination and phosphorylation) and miRNA.DNA methylation could be considered an interesting diagnostic and prognostic marker. But due to limited sample size; and lacking in study the markers with maternal and/or neonatal, the specified outcomes cannot be correlated, more research is needed, furthermore different methods to measure DNA methylation have been utilized. In DNA methylation mainly genes like PDX1 (pancreatic and duodenal homeobox 1), PPARGC1A (Peroxisome proliferator-activated receptor gamma coactivator 1-alpha, GLP1-R played important role.Further GDM is characterized by insulin resistance like in type II DM and various genes are associated with it. Some genes involved in GDM while some are known as inflammatory pathway and involved with maternal metabolism. The IRS1, IGF2BP2, CDKAL1, GCK, TCF7L2, MTNR1B, KCNJ11, KCNQ1, GCKR, HNF4A, SLC30AB, PPARG and FT0 are the genes involved in GDM while LEPR, IL8, TNF $\alpha$, IL6, ADIPOR2, RETN are associated with maternal metabolism the inflammatory pathway gene. The epigenetic and genetic data is important and for that the specific and unique gene to be analyzed and research is needed to limit the number so that diagnosis and screening will be easy and it will provide a better intervention in GDM.

\section{INTRODUCTION}

Diabetes a high blood sugar, if untreated or ignore can causes various health complications. Apart from the two main typesi.e., type I and II the third type is GDM occurs or observed in pregnant women, without history of DM before. So, DM in women can be classified as pre-gestational diabetes mellitus (PGDM) and GDM. The PGDM may be type I or II and should be treated well in

Biosc Biotech Res Comm P-ISSN: 0974-6455 E-ISSN: 2321-4007

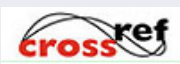

Identifiers and Pagination

Year: 2021 Vol: 14 No (6) Special Issue

Pages: 257-261

This is an open access article under Creative

Commons License Attribn 4.0 Intl (CC-BY).

DOI: $h t t p: / / d x . d o i . o r g / 10.21786 / b b r c / 14.6 .53$ advance of pregnancy if ignored it impose risk to the child and mother which arecongenital malformation, abortions, stillbirth and prenatal mortality. (Int et al.2021, Reece et al.2012, Reece et al.2008, Reece et al.2010, Feig et al.2002, Harris et al.2017 ) Like PGDM the GDM should not be ignored, around 204 million women are affected worldwide and by 2045 it will be projected to increase 308 million. (Diabetes et al. 2012).

It usually develops in second half of pregnancy and may showed various similar causes to foetus as in PGDM, such as prenatal mortality, complications, late neurodevelopment. (kong et al.2019, Ornoy et al.2001) Later years of pregnancy, the GDM women may develop type $2 \mathrm{DM}$ and probably increased chanced and/or high risk to their offspring to develop obesity and Type II DM in early stages of life. (Int et al. 2002) So early diagnosis of PGDM/GDM and optimal control with stringent glycaemic

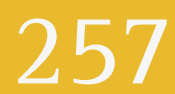


control will decreases the said complications in mother and child, the better foetal survival, normal birth weight and minimum effect on foetus are observed. (Hawthorne et al.2011, Kitzmiller et al.1996, Leguizamon et al.2000, Reece et al.1998, Stévenin et al.2001) It is assumed that the genetic, epigenetic and environmental factors in combination results in GDM.This review highlights epigenetics factors and genes which impact the GDM, maternal and foetal complications.

Gene involved in GDM: The GDM is also characterized by insulin resistance like in type II DM and there are various genes associated with it. Some genes involved in GDM while some are known as inflammatory pathway genes involved with maternal metabolisminflammatory path. The IRS1, IGF2BP2, CDKAL1, GCK, TCF7L2, MTNR1B, KCNJ11, KCNQ1, GCKR, HNF4A, SLC30AB, PPARG and FTO are the genes involved in GDM while LEPR, IL8, TNF $\alpha$, IL6, ADIPOR2, RETN are associated with maternal metabolism the inflammatory pathway gene.

Epigenetic: Genetics is the study of gene and epigeneticis the study of changes in gene expression in DNA sequences. DNA methylation, histone modification, and miRNA gene silencing are the recognizable mechanism of gene expression, and are linked with each other. Disturbances in this complementary system can play a key role in most of the pathogenetic diseases and/or disorders, including GDM as well.

DNA methylation:it is termed as gene silencing, silencing is the position effect i.e., a gene is silenced not to response to a specific environmental signal; it can spread through the large stretches of DNA. Methylation of DNA sequence can inhibit binding of proteins, including the transcriptional machinery and thereby block gene expression. So, in mammal the genes can be kept silent by methylation, and such methylated sequence are often recognized by DNA-binding protein i.e., Methyl $\mathrm{CpG}$ binding protein (MeCP2) which modify nearby chromatin, thus methylated DNA can mark sites where heterochromatin to be formed. (James et al.2017) From the four-base pair of DNA cytosine and adenine can be methylated, where cytosine is widely methylated, methylation of cytosine takes place on 5th position yields 5 methyl cytosine.

In mammals it occurs exclusively occurs or found in $\mathrm{CpG}$ dinucleotide also called CpG island. DNA methylation and or hypermethylation involved in embryonic development, cancer, heart failure, atherosclerosis, exercise, aging, differentiation of B-cell, in brain important for learning and memory. An interesting diagnostic and prognostic marker to intervene GDM could be a DNA methylation. However, the most of the researchstudies carried out with limited sample size and do not correlate the markers with maternal and/or neonatal outcomes considering the GDM. (Ashtankar et al.2020) These limitations make difficult to generalize the meaning of the studies.

PDX1 Gene: It is a protein coding gene (pancreatic and duodenal homeobox 1) attributed with 283 amino acid with molecular mass of 30771 D.It is a insulin promoter factor 1 a transcription factor in gene cluster of ParaHox., also known as insulin promoter factor 1 and is important in development of pancreas in human and all associate vertebrates. (Ashfaque et al.2020, Brooke et al.1998).

For regulation of $\beta$ - cell development 6 super-pathways and 36 genes are involved including PDX1. There are 37 different disorders observed associated with the PDX1 gene, in which mainly pancreatic agenesis, type I \& II DM etc. The protein encoded by this gene is a transcriptional activator of several genes which includes insulin, somatostatin, islet amyloid polypeptide, glucokinase and type 2 glucose transporter. It is involved in early development of the pancreas and has a major role in glucose-dependent regulation of insulin gene expression. So, defects in this gene cause pancreatic agenesis, which can lead to early-onset of IDDM. (Biol et al.2005).

At molecular level the function for PDX1 gene is to activate insulin, somatostatin etc, the complex PDX1:PBX1b: MEIS2b of acinar cells activates the ELA1 enhancer so the complex gets bind to the enhancer B, which actually specifies the early pancreatic epithelium, proliferation and differentiation during development. Joshua Francis et al studies the methylation of PDX1 gene, suggest that it may be methylated by SETD7 in vitro. The study revel the disruption of PDX 1 expression during development resulting in undeveloped pancreas. (Chem et al.2005)Study describe the role PDX1 in maintenance of H3-K4 methylation, it is actually associated with H3K4 methyltransferase to insulin gene.

Ppargc1a: Peroxisome proliferator-activated receptor gamma coactivator 1 -alpha $(\mathrm{PGC}-1 \alpha)$ is a protein encoded by PPARGC1A gene in humans. (Inamdar et al. 2020) The energy metabolism has been regulated by this protein which is by this gene,interact with PPAR gamma and permits the interaction with multiple transcription factors as it is a transcriptional coactivator. Further it interacts and regulates c-AMP response element binding protein (CREB) and nuclear respiratory factors (NRFs). Thereby, providing link between mitochondrial biogenesis \& physiological external stimuli to regulatedetermination of muscle fiber type.

Apart from this it controls blood pressure, cholesterol homeostasis and obesity. (Jankar et al. 2020)Impaired insulin secretion has been observed if down-regulation of PGC- $1 \alpha$ which leads to improper mitochondrial functioning and disturbances in ATP production. (Unnikrishnan et al. 2020) The various metabolic processes like glycogenolysis, gluconeogenesis, fatty acid oxidation, oxidative phosphorylation, glucose transport has been control through this gene, further this gene is expressed predominantly in tissues with high energy demand including the skeletal muscle, pancreas, and liver. (Ahmed et al. 2020) The down regulation of this gene expression results reduced expression of insulin mRNA and there by decreases insulin secretion. 
GLP1-R: This gene is attributes 463 amino acid with molecular mass $53026 \mathrm{Da}$, a 7-transmembrane protein which function as receptor and stimulates glucoseinduced insulin.

The cell surfaced receptor plays very important role in insulin secretion through cell signalling cascades. This cascade activates the adenylyl cyclise and increased intracellular c-AMP.Polymorphism in this gene is associated with diabetes. The GLP-1R is a key pharmacological target to treat type II DM. Teresa Buenaventura et al studies the important process of intracellular signalling and clathrin-mediated endocytosis, which is very dynamically regulated. (Teresa Buenaventura et al. 2019)In another study suggest that GLP-1 like peptides is important as if lower level of these peptides may produce abnormality in glucose regulation following pregnancy. (Ambad et al. 2020).

Histone Modifications: These are the positively charged protein containing core histones which are $\mathrm{H} 2 \mathrm{~A}, \mathrm{H} 2 \mathrm{~B}$, H3 \& H4 and linkers H1 \& H5. (Ranjit et al.2020, Guo et al.2020) The main mechanism is to regulate the structure of chromatin which actually occurs on lysine, arginine, serine, tyrosine and threonine amino acids which further influence the transcriptional activity. (Jezek et al. 2019) There is epigenetic writer, erasers and readers which actually mediate histone modification, acetyltransferas es, methyltransferases are writer, while deacetylases \& demethylases are eraser.Histone modification mainly includes, its methylation, acetylation, crotonylation, Ubiquitination and phosphorylation. Thesemodifications occur on respective amino acids, mainly the lysine and arginine are widely studied. (Seshan et al.2021, Tan et al.2011, Fontecha et al.2018).

Methylation on Histone: primarily lysine and arginine amino acid residues are methylated. (Klose et al.2007) The mono, di and tri methylated amino acid residues are the three forms of histone methylation which increase the complexity of PTM. Considering Lysine its methylation is catalysed by histone methyltransferases while lysine demethylases remove methyl groups from histone known as demethylation of histone. (Hadke et al. 2021, Metzger et al.2005) The histone methylation is reversible modification and dynamic regulate by histone methyltransferases and lysine demethylases. This modification is the most stable PTM and showed considerable studies and/or research observations that it contributedinno of pathogenesis including GDM.

Acetylation of Histone: Addition of acetyl group on the lysine of histone through histone acetyltransferases (HATs). Acetyl group on histone reduces the -ve charge of DNA to made easy accessibility of chromatin for transcription factors and coactivators. (Xu et al. 2019) The histone deacetylation is catalysed by histone deacetylases which regulate transcriptional repression. Crotonylation of Histone: the crotonyl group is transferred to lysine residue, it can activate gene transcription in a gene or environment dependent manner. (Tan et al.2011).
miRNA: These arenon-coding and regulates the gene expression by inhibition or degradation its translation. (Michels et al. 2012) These are associated with insulin secretion, resistance and inflammation and having different circulating levels in type II diabetic and nondiabetic patients. (Zhao et al. 2010) The level of miRNA was examined in pregnant women showed decreased expression of it which includes miR-29a, miR-132 and miR222. (Zhao et al. 2011) The miR29a regulates the glucose homeostasis and its over-expression downregulates the gluconeogenesis, the miR-132 involved in trophoblast expansion its down-regulation gives abnormal development, while the miR222 regulated the cell cycle. (Zhou et al.2019, Zhu et al.2015).

\section{CONCLUSION}

In conclusion we can confirm that the epigenetic, genetic and environmental factors play important role in onset of GDM, and there are some genes which are unique to pregnancy. Epigenetic changes which include the DNA methylation, various modification on histone and miRNA gene have been identified in gestational diabetes mellitus. But in clinical practice the nutritional and lifestyle indications has been noted or suggested to the patients to reduce the epigenetic or genetic impact on mother and child as well. Still exercise with proper balance nutritious diet showed positive effect. Apart from this the epigenetic and genetic data is important and for that the specific and unique gene to be analyzed part of it we discussed in this review, but further research is needed to limit the number so the diagnosis and screening will be easy and it will provide a better intervention in GDM.

\section{REFERENCES}

Ahmed, S.A.H., Ansari, S.A., Mensah-Brown, E.P. and Emerald, B.S., 2020. The role of DNA methylation in the pathogenesis of type 2 diabetes mellitus. Clinical Epigenetics, 12(1), pp.1-23.

Ambad, R., Nagpure, S., Subramaniam Seshan, D. and Nagrale, N., 2021. Therapeutic Potential Of Yoga Practices In Management Of Diabetes. European Journal of Molecular \&t Clinical Medicine, 8(1), pp.174-182.

Ashfaque, A.R.M., Khanam, N., Khan, F., Waghmare, R.N. and Joshi, S.K., 2020. Assessment of Self-Care Practices among Type 2 Diabetes Patients at a Tertiary Care Hospital--A Cross-Sectional Study. Journal of Evolution of Medical and Dental Sciences, 9(36), pp.2630-2636. Ashtankar, P.V., Sawarkar, P., Patil, M. and Singh, K., Comparativeevaluation of the efficacy of Panchatikta Panchaprasutik Niruha Vastiand lifestyle modification in Prediabetes (Prameha Poorvaroopa).

Bajaj, S., 2017. Women and Diabetes: They have a right to a healthy future!. JPMA. The Journal of the Pakistan Medical Association, 67(11), pp.1643-1645.

Brooke, N.M., Garcia-Fernàndez, J. and Holland, P.W., 1998. The ParaHox gene cluster is an evolutionary sister of the Hox gene cluster. Nature, 392(6679), pp.920922.

Buenaventura, T., Bitsi, S., Laughlin, W.E., Burgoyne, 
T., Lyu, Z., Oqua, A.I., Norman, H., McGlone, E.R., Klymchenko, A.S., Corrêa Jr, I.R. and Walker, A., 2019. Agonist-induced membrane nanodomain clustering drives GLP-1 receptor responses in pancreatic beta cells. PLoS biology, 17(8), p.e3000097.

Christianson, D.B., Anderson, S.E., He, L.W., Salesin, D.H., Weld, D.S. and Cohen, M.F., 1996, August. Declarative camera control for automatic cinematography. In AAAI/ IAAI, Vol. 1 (pp. 148-155).

Christianson, D.B., Anderson, S.E., He, L.W., Salesin, D.H., Weld, D.S. and Cohen, M.F., 1996, August. Declarative camera control for automatic cinematography. In AAAI/ IAAI, Vol. 1 (pp. 148-155).

Dalfrà, M.G., Burlina, S., Del Vescovo, G.G. and Lapolla, A., 2020. Genetics and Epigenetics: New Insight on Gestational Diabetes Mellitus. Frontiers in Endocrinology, 11, p.936.

Feig, D.S. and Palda, V.A., 2002. Type 2 diabetes in pregnancy: a growing concern. The Lancet, 359(9318), pp.1690-1692.

Fontecha-Barriuso, M., Martin-Sanchez, D., RuizAndres, 0., Poveda, J., Sanchez-Niño, M.D., ValinoRivas, L., Ruiz-Ortega, M., Ortiz, A. and Sanz, A.B., 2018. Targeting epigenetic DNA and histone modifications to treat kidney disease. Nephrology dialysis transplantation, 33(11), pp.1875-1886.

Francis, J., Chakrabarti, S.K., Garmey, J.C. and Mirmira, R.G., 2005. Pdx-1 links histone H3-Lys-4 methylation to RNA polymerase II elongation during activation of insulin transcription. Journal of Biological Chemistry, 280(43), pp.36244-36253.

Gerstein, H.C., Mann, J.F., Yi, Q., Zinman, B., Dinneen, S.F., Hoogwerf, B., Hallé, J.P., Young, J., Rashkow, A., Joyce, C. and Nawaz, S., 2001. Albuminuria and risk of cardiovascular events, death, and heart failure in diabetic and nondiabetic individuals. Jama, 286(4), pp.421-426.

Guo, C., Dong, G., Liang, X. and Dong, Z., 2019. Epigenetic regulation in AKI and kidney repair: mechanisms and therapeutic implications. Nature Reviews Nephrology, 15(4), pp.220-239.

Harris, B.S., Bishop, K.C., Kemeny, H.R., Walker, J.S., Rhee, E. and Kuller, J.A., 2017. Risk factors for birth defects. Obstetrical \& gynecological survey, 72(2), pp.123-135.

Hawthorne, G., 2011. Maternal complications in diabetic pregnancy. Best practice \& research Clinical obstetrics Et gynaecology, 25(1), pp.77-90.

Inamdar, S.A., Agarwal, H., Acharya, S. and Inamdar, A., 2020. Coexistence of hypertriglyceredemia and hypercholesterolemia with gestational diabetes mellitus in pregnancy: A case report. Medical Science, 24(102), pp.594-598.

Jankar, J.S., Harley, K.N., Mohod, K.M. and Babar, V.Y., Association of Urinary Albumin with HbA1c Levels in Subjects of Type 2 Diabetes Mellitus in Central India. Jezek, M. and Green, E.M., 2019. Histone modifications and the maintenance of telomere integrity. Cells, 8(2), p.199.

Kitzmiller, J.L. and Combs, C.A., 1996. Diabetic nephropathy and pregnancy. Obstetrics and Gynecology Clinics, 23(1), pp.173-203.

Klose, R.J. and Zhang, Y., 2007. Regulation of histone methylation by demethylimination and demethylation. Nature reviews Molecular cell biology, 8(4), pp.307318.

Kong, L., Nilsson, I.A., Gissler, M. and Lavebratt, C., 2019. Associations of maternal diabetes and body mass index with offspring birth weight and prematurity. JAMA pediatrics, 173(4), pp.371-378.

Latchoumi, T.P., Ezhilarasi, T.P. and Balamurugan, K., 2019. Bio-inspired weighed quantum particle swarm optimization and smooth support vector machine ensembles for identification of abnormalities in medical data. SN Applied Sciences, 1(10), pp.1-10.

Leguizamon, G. and Reece, E.A., 2000. Effect of medical therapy on progressive nephropathy: influence of pregnancy, diabetes and hypertension. The Journal of Maternal-Fetal Medicine, 9(1), pp.70-78.

Li, J., Yuan, D., Wang, P., Wang, Q., Sun, M., Liu, Z., Si, H., Xu, Z., Ma, Y., Zhang, B. and Pei, L., 2021. Cotton pan-genome retrieves the lost sequences and genes during domestication and selection. Genome biology, 22(1), pp.1-26.

Lowe, L.P., Metzger, B.E., Dyer, A.R., Lowe, J., McCance, D.R., Lappin, T.R., Trimble, E.R., Coustan, D.R., Hadden, D.R., Hod, M. and Oats, J.J., 2012. Hyperglycemia and Adverse Pregnancy Outcome (HAP0) Study: associations of maternal A1C and glucose with pregnancy outcomes. Diabetes care, 35(3), pp.574-580.

Metzger, E., Wissmann, M. and Yin, N., Mü ller JM, Schneider R, Peters AHFM, Gü nther T, Buettner R, Schü le R. 2005. LSD1 demethylates repressive histone marks to promote androgen-receptor-dependent transcription. Nature, 437, pp.436-439.

Mosavat, M., Omar, S.Z., Jamalpour, S. and Tan, P.C., 2020. Serum Glucose-Dependent Insulinotropic Polypeptide (GIP) and Glucagon-Like Peptide-1 (GLP-1) in association with the Risk of Gestational Diabetes: A Prospective Case-Control Study. Journal of diabetes research, 2020.

Ornoy, A., Becker, M., Weinstein-Fudim, L. and Ergaz, Z., 2021. Diabetes during Pregnancy: A Maternal Disease Complicating the Course of Pregnancy with Long-Term Deleterious Effects on the Offspring. A Clinical Review. International Journal of Molecular Sciences, 22(6), p. 2965.

Ornoy, A., Ratzon, N., Greenbaum, C., Wolf, A. and Dulitzky, M., 2001. School-age children born to diabetic mothers and to mothers with gestational diabetes exhibit a high rate of inattention and fine and gross motor impairment. Journal of Pediatric Endocrinology and Metabolism, 14(Supplement), pp.681-690.

Reece, E.A., 2008. Perspectives on obesity, pregnancy 
and birth outcomes in the United States: the scope of the problem. American journal of obstetrics and gynecology, 198(1), pp.23-27.

Reece, E.A., 2010. The fetal and maternal consequences of gestational diabetes mellitus. The journal of maternalfetal \&t neonatal medicine, 23(3), pp.199-203.

Reece, E.A., 2012. Diabetes-induced birth defects: what do we know? What can we do?. Current diabetes reports, 12(1), pp.24-32.

Reece, E.A., Leguizamon, G. and Homko, C., 1998. Pregnancy performance and outcomes associated with diabetic nephropathy. American journal of perinatology, 15(07), pp.413-421.

Rodrigues, P. and Rosa, P.J., 2019. Eye-tracking as a research methodology in educational context: A spanning framework.

Stevenin, C., Collet, C., White, C. and Marre, M., 2001. Management of diabetic nephropathy before and during pregnancy. Diabetes \& metabolism, 27(4 Pt 2), pp.S42-7.

Unnikrishnan, B., Singh, A., Rathi, P., Bhat, S.K., Ravishankar, N., Nayak, P.H. and Praveen, O., 2020. Risk factors of gestational diabetes mellitus: A hospital-based pairmatched case-control study in coastal South India. South African Journal of Obstetrics and Gynaecology,
26(1), pp.13-17.

Wagh, S.P., Bhagat, S.P., Bankar, N. and Jain, K., 2020. Role of Vitamin-C Supplementation in Type II Diabetes Mellitus. Int J Cur Res Rev, 2020.

$\mathrm{Xu}$, L., Natarajan, R. and Chen, Z., 2019. Epigenetic risk profile of diabetic kidney disease in high-risk populations. Current diabetes reports, 19(3), p.9.

Zhao, C., Dong, J., Jiang, T., Shi, Z., Yu, B., Zhu, Y., Chen, D., Xu, J., Huo, R., Dai, J. and Xia, Y., 2011. Early second-trimester serum miRNA profiling predicts gestational diabetes mellitus. PloS one, 6(8), p.e23925.

Zhao, H., Guan, J., Lee, H.M., Sui, Y., He, L., Siu, J.J., Patty, P.P., Tong, P.C., Lai, F.M. and Chan, J.C., 2010. Upregulated pancreatic tissue microRNA-375 associates with human type 2 diabetes through $\otimes$-cell deficit and islet amyloid deposition. Pancreas, 39(6), pp.843-846. Zhou, X., Xiang, C. and Zheng, X., 2019. miR-132 serves as a diagnostic biomarker in gestational diabetes mellitus and its regulatory effect on trophoblast cell viability. Diagnostic pathology, 14(1), pp.1-7.

Zhu, H. and Leung, S.W., 2015. Identification of microRNA biomarkers in type 2 diabetes: a metaanalysis of controlled profiling studies. Diabetologia, 58(5), pp.900-911. 